Transactions of the American Fisheries Society 144:124-139, 2015

(C) American Fisheries Society 2015

DOI: $10.1080 / 00028487.2014 .968293$

\title{
Supplement: Deciphering Hatchery Stock Influences on Wild Populations of Vermont Lake
} Trout

Supplementary Figure S.1. Allele frequency distributions generated in the Mode Shift test (program BOTTLENECK; Cornuet and Luikart 1997) for northeastern Vermont Lake Trout populations and Lake Champlain hatchery broodstock using all 11 microsatellite loci (top panel) and excluding locus Saz72im due to possible null alleles (bottom panel). The allele frequency class of 0.1 signifies an abundance of rare alleles in all populations.
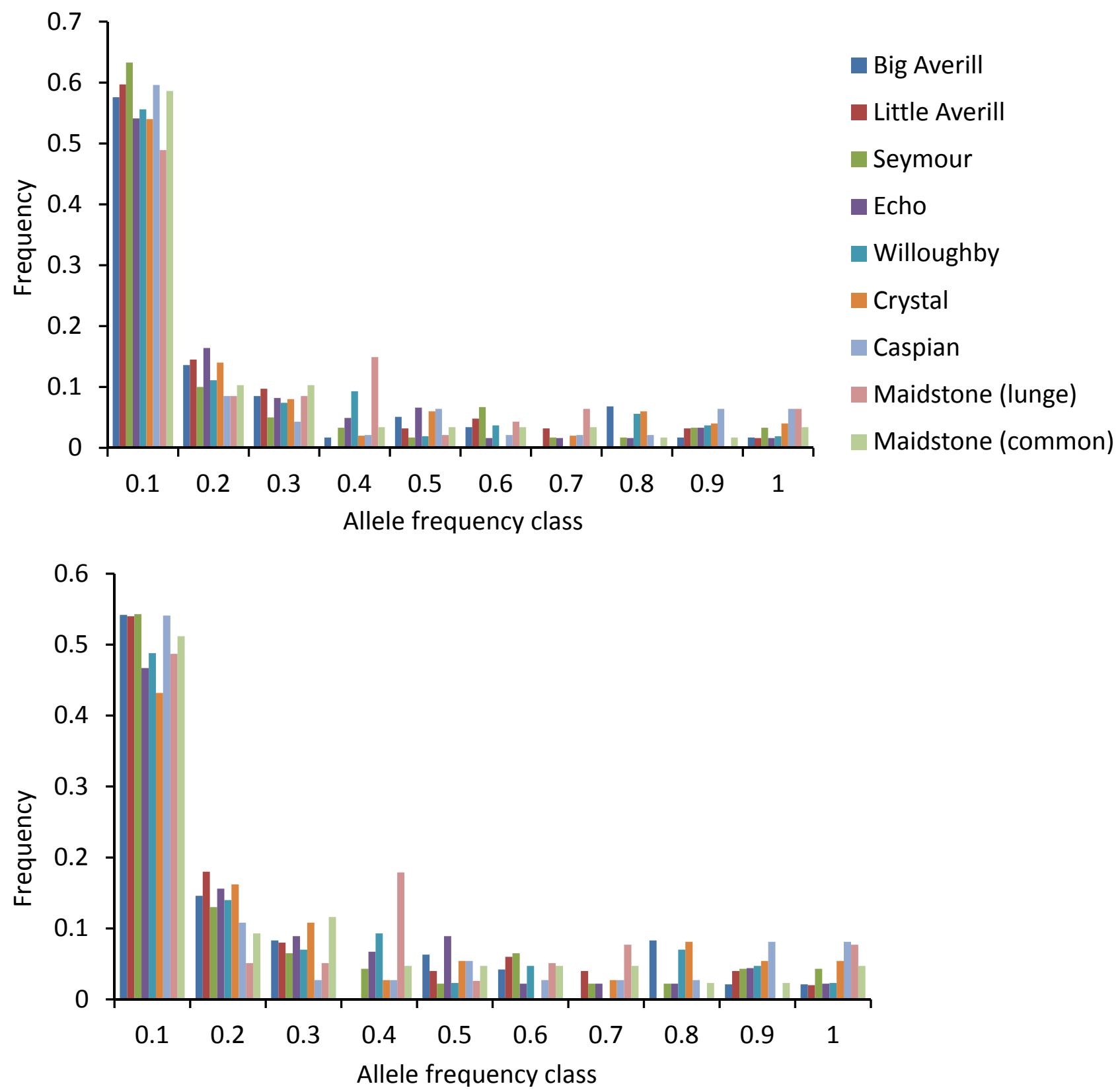
Supplementary Figure S.2. Results of Bayesian genetic population structure analysis (program STRUCTURE; Pritchard et al. 2000; Falush et al. 2007; Hubisz et al. 2009) of 10 Vermont Lake Trout populations. To determine the number of populations (K), plots of the mean of estimated natural log probably of $K$ (left panel) and delta $K$ (right panel) were constructed using the Evanno method (Evanno et al. 2005) in program STRUCTURE HARVESTER Web 0.6.92 (Earl and von Holdt 2012).
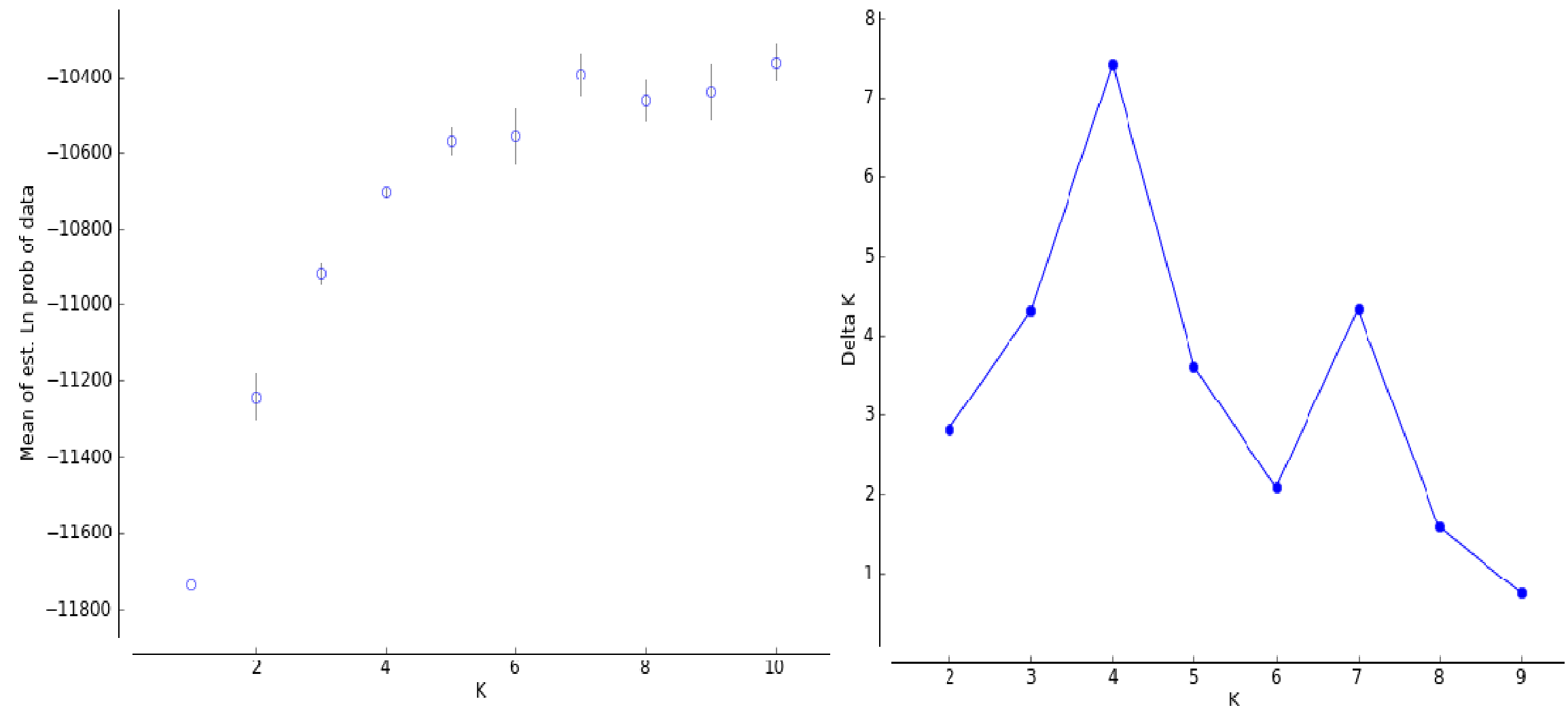
Supplementary Figure S.3. Inference of the number of genetic populations, $K$, using discriminant analysis of principal components (Jombart et al. 2010) with the R package (R Development Core Team 2012), program ADEGENET 1.2.8 (Jombart 2008). The figures show (A) the Bayesian information criterion (BIC) plot, (B) the comparison of fit of inferred clusters to actual localities, and the histograms of (C) the first and (D) the second discriminant functions of the discriminant analysis of principal components.

(A)

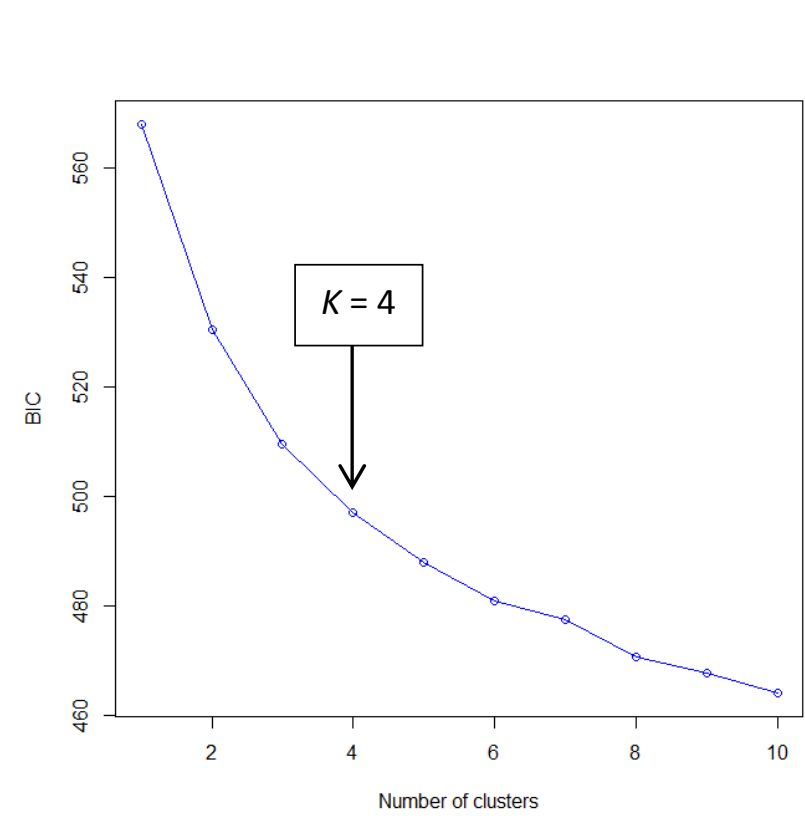

(C)

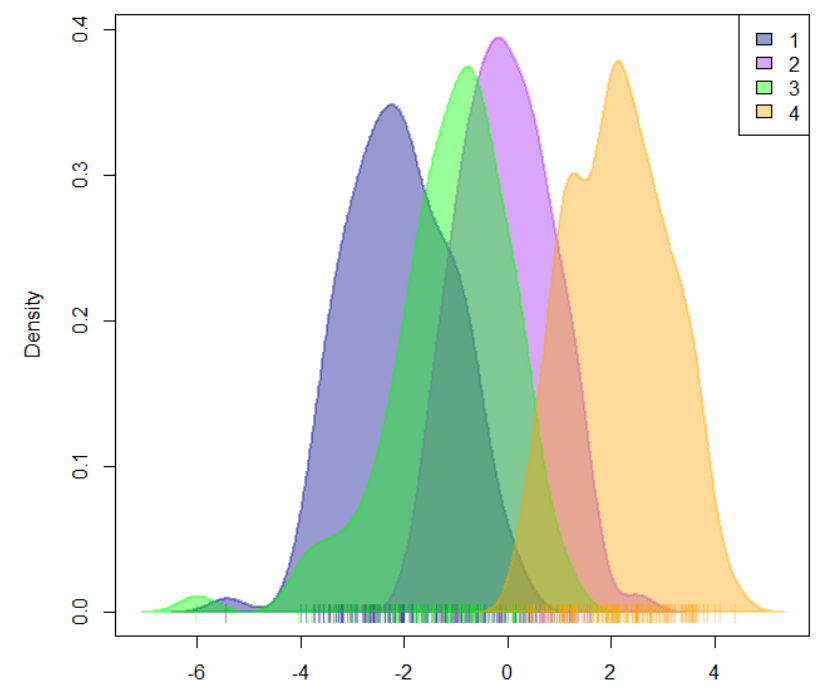

(B)

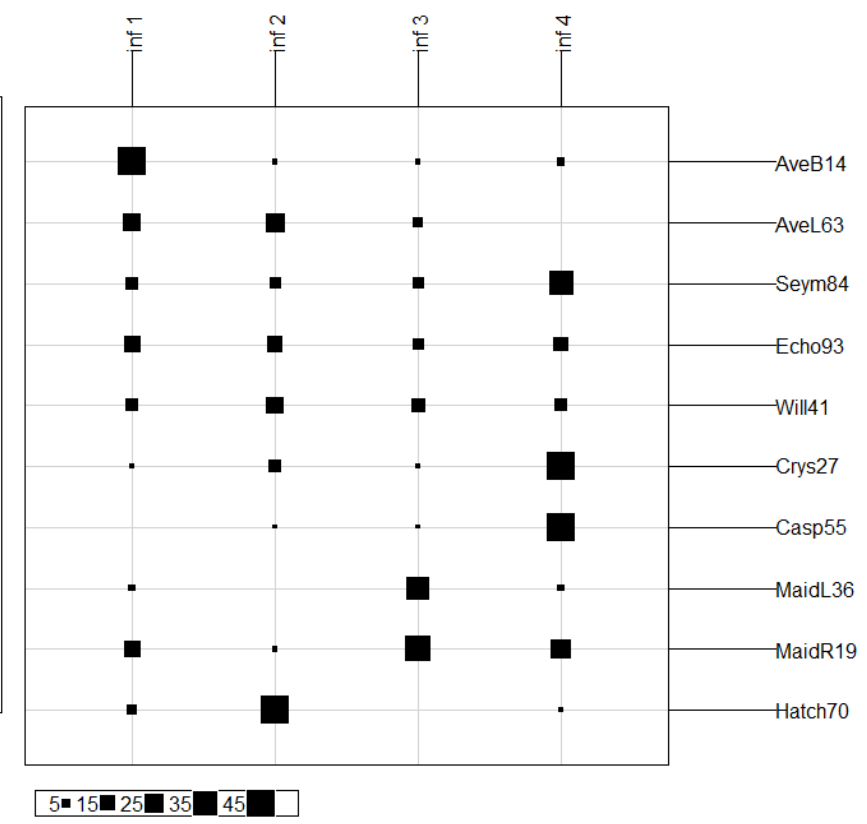

(D)

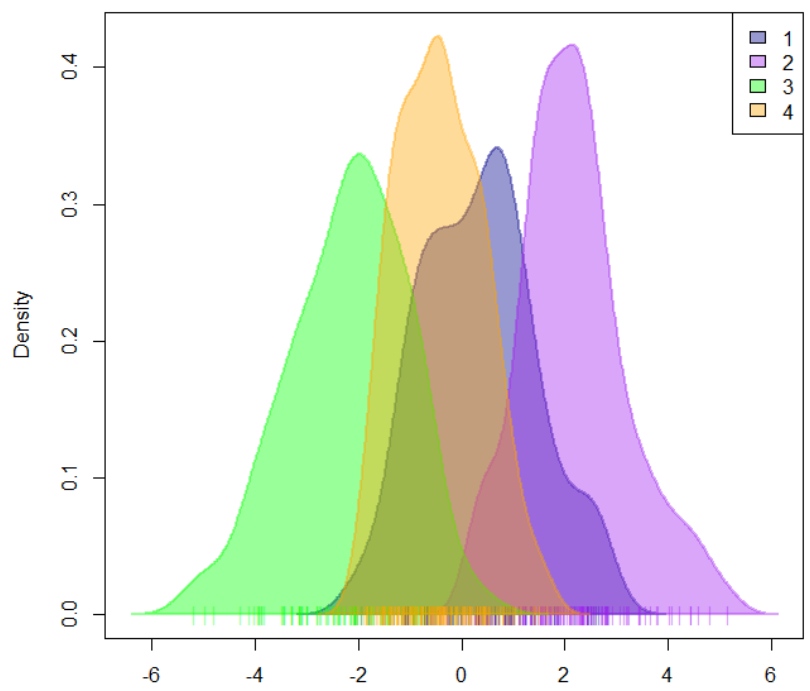


Supplementary Figure S.4. (A) Triangle plot and corresponding (B) Bayesian clustering bar plot generated in program STRUCTURE (Pritchard et al. 2000; Falush et al. 2003; Hubisz et al. 2009), showing membership of individuals (colored dots) to each population using the "test for migrants" ancestry model set at the present generation, $K=7$ populations, two generations back, and maximum 5\% prior migration. The seven individuals with a high probably of ancestry in the Lake Champlain hatchery strain are represented by numbers 1-7. Population color codes do not correspond among figures.

(A)

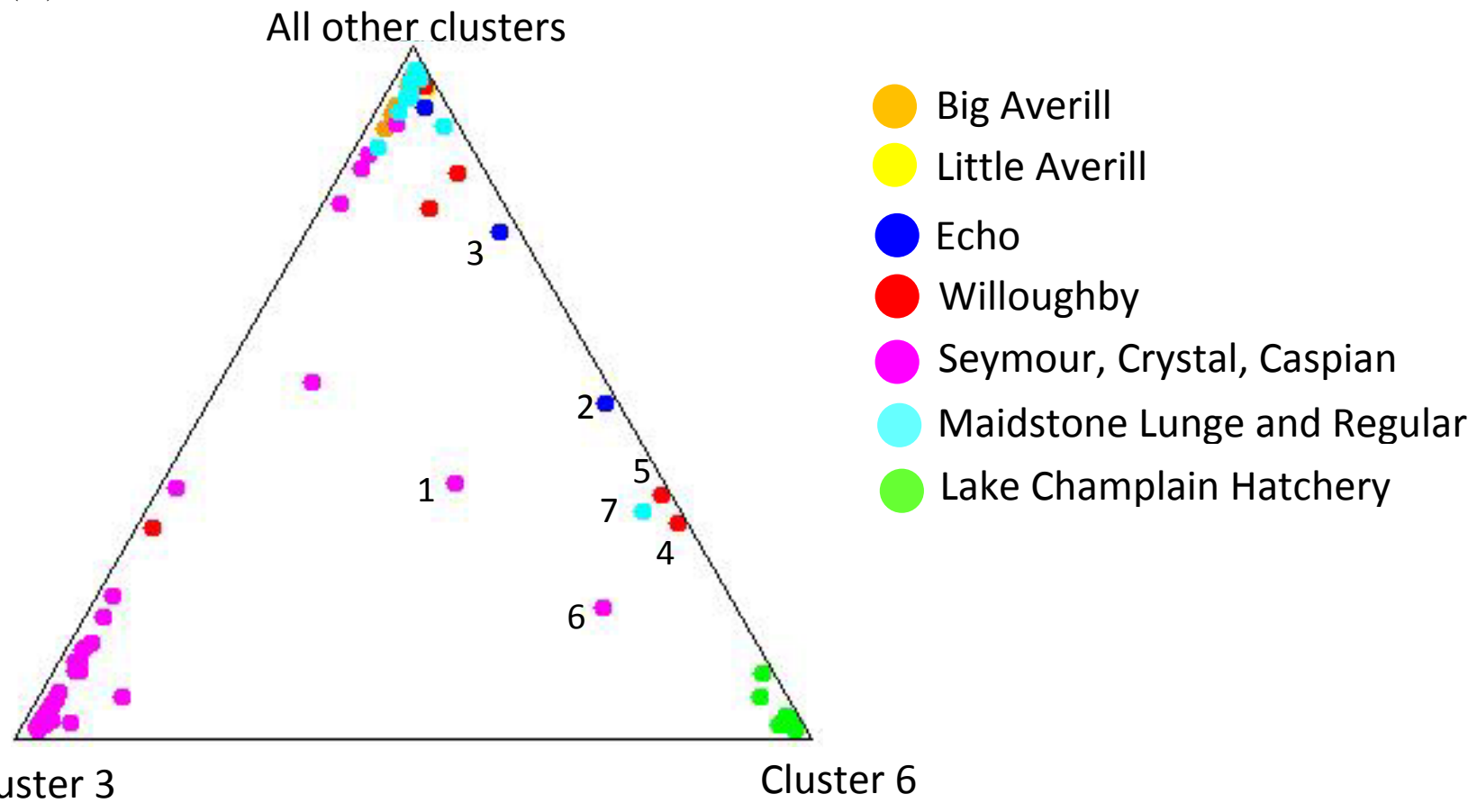

(B)

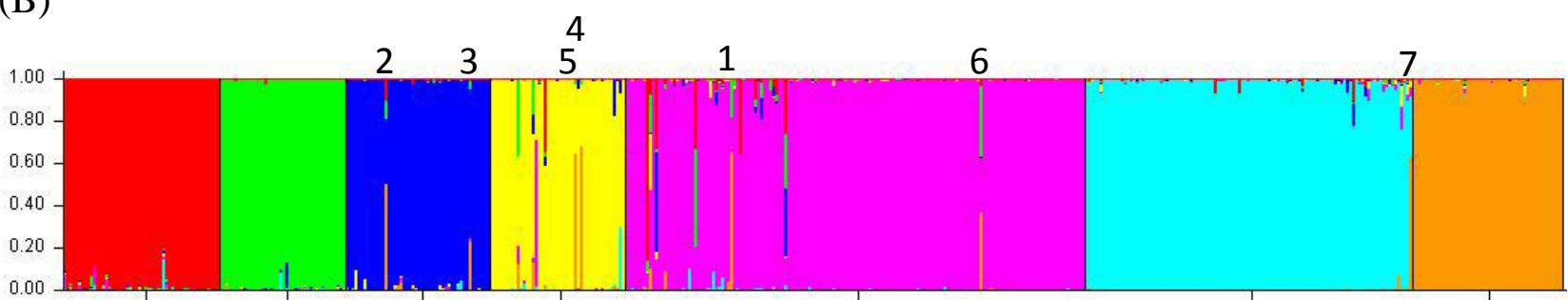

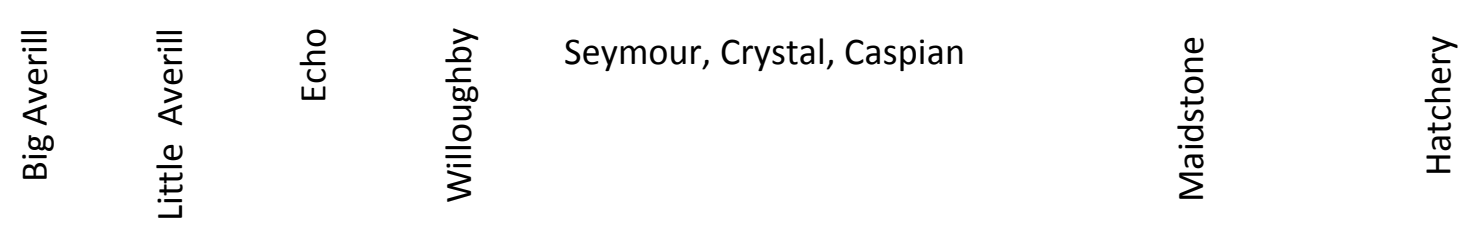


Supplementary Table S.1. Allelic frequencies for 11 microsatellite loci at 10 Lake Trout sampling populations in the Vermont Northeast Kingdom as calculated using ARLEQUIN (Excoffier and Lischer 2010). Private alleles for each population are shaded gray.

\begin{tabular}{|c|c|c|c|c|c|c|c|c|c|c|c|}
\hline \multirow[t]{2}{*}{$\begin{array}{l}\text { Locus } \\
\text { name }\end{array}$} & \multirow[t]{2}{*}{$\begin{array}{l}\text { Allele } \\
\text { size } \\
\text { (bp) }\end{array}$} & \multicolumn{10}{|c|}{ Location } \\
\hline & & 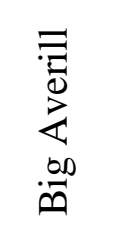 & 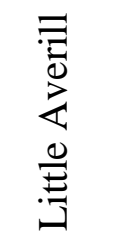 & 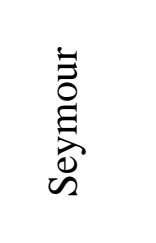 & 总 & $\begin{array}{l}\overrightarrow{0} \\
\text { 离 } \\
\overrightarrow{0} \\
\overrightarrow{7} \\
\overrightarrow{3}\end{array}$ & 㺃 & 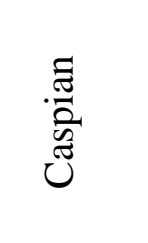 & 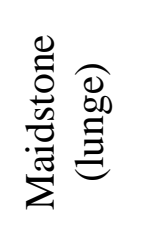 & 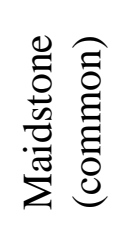 & 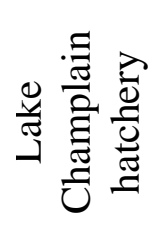 \\
\hline \multirow[t]{5}{*}{ Ssa85 } & 128 & & 0.036 & & 0.021 & & & & & & \\
\hline & 136 & 0.260 & 0.512 & 0.519 & 0.448 & 0.522 & 0.800 & 0.809 & 0.338 & 0.412 & 0.480 \\
\hline & 138 & 0.019 & 0.024 & 0.009 & & 0.022 & & & & & 0.030 \\
\hline & 140 & 0.702 & 0.429 & 0.472 & 0.531 & 0.456 & 0.200 & 0.181 & 0.662 & 0.574 & 0.490 \\
\hline & 142 & 0.019 & & & & & & 0.011 & & 0.014 & \\
\hline \multirow[t]{13}{*}{ Sco202 } & 150 & 0.019 & 0.012 & & 0.021 & 0.012 & & & & & 0.040 \\
\hline & 154 & & 0.024 & & & 0.012 & & & & & \\
\hline & 158 & & 0.024 & 0.009 & & & 0.010 & & 0.015 & & \\
\hline & 162 & 0.029 & 0.095 & 0.046 & & 0.070 & 0.067 & 0.033 & 0.015 & 0.014 & \\
\hline & 166 & 0.154 & 0.119 & 0.287 & 0.219 & 0.360 & 0.346 & 0.467 & 0.338 & 0.279 & 0.340 \\
\hline & 170 & 0.212 & 0.238 & 0.111 & 0.240 & 0.209 & 0.260 & 0.322 & 0.206 & 0.179 & 0.320 \\
\hline & 174 & 0.183 & 0.167 & 0.306 & 0.281 & 0.163 & 0.192 & 0.156 & 0.309 & 0.286 & 0.140 \\
\hline & 178 & 0.202 & 0.155 & 0.130 & 0.063 & 0.070 & 0.106 & 0.011 & 0.074 & 0.150 & 0.080 \\
\hline & 182 & 0.125 & 0.060 & 0.074 & 0.156 & 0.093 & 0.019 & & 0.015 & 0.086 & 0.040 \\
\hline & 186 & 0.058 & 0.048 & 0.019 & & 0.012 & & & 0.029 & 0.007 & 0.040 \\
\hline & 190 & 0.019 & 0.060 & 0.019 & 0.010 & & & & & & \\
\hline & 194 & & & & & & & 0.011 & & & \\
\hline & 206 & & & & 0.010 & & & & & & \\
\hline
\end{tabular}




\begin{tabular}{|c|c|c|c|c|c|c|c|c|c|c|c|}
\hline \multirow[t]{2}{*}{$\begin{array}{l}\text { Locus } \\
\text { name }\end{array}$} & $\begin{array}{l}\text { Allele } \\
\text { size } \\
\text { (bp) }\end{array}$ & \multicolumn{10}{|c|}{ Location } \\
\hline & & 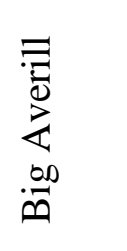 & 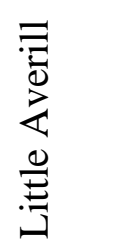 & 刍 & 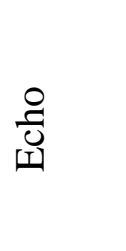 & $\begin{array}{l}\text { 合 } \\
\frac{0}{000} \\
0 \\
0 \\
3 \\
3\end{array}$ & $\sum_{0}^{\frac{\pi}{4}}$ & 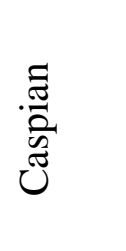 & 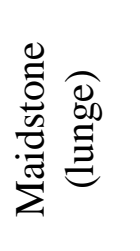 & 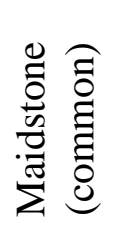 & 烰 \\
\hline & 150 & & & & & & & & 0.043 & 0.028 & \\
\hline & 152 & & & & & & & & & 0.014 & \\
\hline & 160 & 0.250 & 0.145 & 0.343 & 0.466 & 0.089 & 0.449 & 0.415 & 0.243 & 0.282 & 0.234 \\
\hline & 166 & 0.058 & & 0.009 & & & & & & & \\
\hline & 168 & & 0.026 & & & & & & & & \\
\hline & 170 & 0.529 & 0.539 & 0.519 & 0.420 & 0.800 & 0.439 & 0.521 & 0.614 & 0.620 & 0.415 \\
\hline & 172 & 0.048 & 0.026 & 0.065 & 0.034 & & 0.020 & & & 0.007 & \\
\hline & 174 & 0.115 & 0.197 & 0.056 & 0.080 & 0.100 & & 0.043 & 0.100 & 0.049 & 0.298 \\
\hline & 176 & & 0.013 & & & 0.011 & & 0.011 & & & 0.021 \\
\hline & 178 & & 0.053 & 0.009 & & & 0.020 & 0.011 & & & 0.032 \\
\hline & 188 & & & & & & 0.061 & & & & \\
\hline
\end{tabular}

\begin{tabular}{|c|c|c|c|c|c|c|c|c|c|c|c|}
\hline \multirow[t]{2}{*}{ OneU9 } & 242 & 0.012 & & & & & & \multirow[b]{2}{*}{0.985} & \multirow[b]{2}{*}{0.586} & \multirow[b]{2}{*}{0.750} & \multirow[b]{2}{*}{1.000} \\
\hline & 246 & 0.791 & 0.872 & 0.870 & 0.840 & 0.872 & 0.913 & & & & \\
\hline & 248 & 0.198 & 0.128 & 0.130 & 0.149 & 0.128 & 0.087 & & 0.414 & 0.250 & \\
\hline & 250 & & & & 0.011 & & & & & & \\
\hline & 264 & & & & & & & 0.015 & & & \\
\hline \multirow[t]{5}{*}{ Ogo1 } & 146 & 0.192 & 0.079 & 0.574 & 0.323 & 0.511 & 0.615 & 0.696 & 0.629 & 0.412 & 0.290 \\
\hline & 150 & 0.019 & 0.013 & & 0.010 & & & & & & \\
\hline & 152 & 0.769 & 0.697 & 0.287 & 0.375 & 0.367 & 0.135 & 0.054 & 0.343 & 0.385 & 0.230 \\
\hline & 154 & 0.019 & 0.171 & 0.139 & 0.292 & 0.100 & 0.250 & 0.250 & 0.029 & 0.196 & 0.480 \\
\hline & 156 & & 0.039 & & & 0.022 & & & & 0.007 & \\
\hline
\end{tabular}




\begin{tabular}{|c|c|c|c|c|c|c|c|c|c|c|c|}
\hline \multirow[t]{2}{*}{$\begin{array}{l}\text { Locus } \\
\text { name }\end{array}$} & \multirow[t]{2}{*}{$\begin{array}{l}\text { Allele } \\
\text { size } \\
\text { (bp) }\end{array}$} & \multicolumn{10}{|c|}{ Location } \\
\hline & & 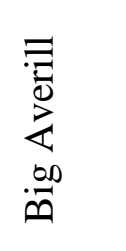 & 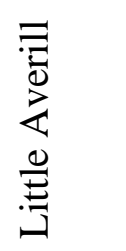 & 当 & 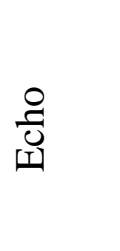 & $\begin{array}{l}\frac{1}{0} \\
\frac{1}{00} \\
0 \\
0 \\
3 \\
3\end{array}$ & $\underset{0}{\frac{\pi}{5}}$ & 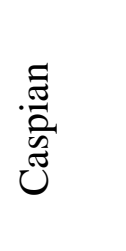 & 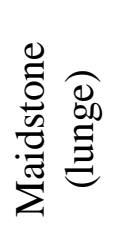 & 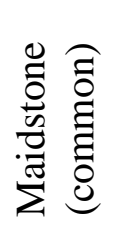 & 冚 \\
\hline \multirow[t]{9}{*}{ Sfo12 } & 232 & & & & 0.010 & 0.222 & & & & & \\
\hline & 246 & & & & & & & 0.021 & & & \\
\hline & 250 & & & & 0.010 & & & & & & \\
\hline & 252 & & 0.036 & 0.009 & & 0.011 & 0.029 & & 0.014 & 0.007 & 0.030 \\
\hline & 254 & 0.106 & 0.083 & 0.028 & 0.052 & 0.011 & 0.029 & & & 0.047 & 0.150 \\
\hline & 256 & 0.865 & 0.881 & 0.963 & 0.927 & 0.756 & 0.942 & 0.979 & 0.957 & 0.946 & 0.800 \\
\hline & 258 & 0.029 & & & & & & & & & \\
\hline & 260 & & & & & & & & & & 0.020 \\
\hline & 262 & & & & & & & & 0.029 & & \\
\hline \multirow[t]{7}{*}{ SfoB52 } & 216 & & & 0.009 & 0.011 & & & & 0.048 & 0.035 & \\
\hline & 218 & 0.461 & 0.224 & 0.222 & 0.322 & 0.284 & 0.077 & 0.011 & 0.371 & 0.310 & 0.150 \\
\hline & 220 & 0.010 & 0.092 & 0.056 & 0.022 & 0.011 & & 0.022 & & & 0.290 \\
\hline & 222 & & & & & & 0.010 & & & & \\
\hline & 224 & 0.069 & 0.474 & 0.083 & 0.167 & 0.330 & 0.115 & 0.133 & 0.032 & 0.028 & 0.270 \\
\hline & 228 & 0.461 & 0.211 & 0.620 & 0.478 & 0.364 & 0.798 & 0.833 & 0.548 & 0.627 & 0.090 \\
\hline & 232 & & & 0.009 & & 0.011 & & & & & 0.200 \\
\hline
\end{tabular}

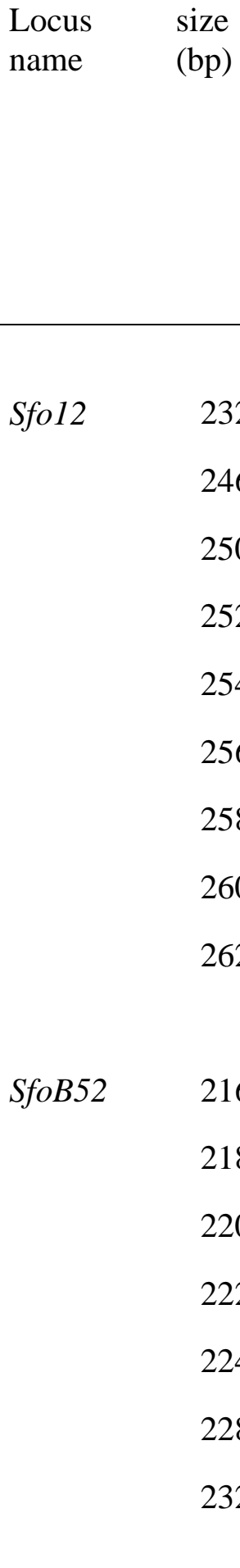

$\begin{array}{lllllllllll}102 & 0.404 & 0.274 & 0.120 & 0.135 & 0.144 & 0.048 & 0.064 & 0.043 & 0.068 & 0.370\end{array}$ $\begin{array}{lllllllllll}105 & 0.529 & 0.595 & 0.731 & 0.688 & 0.756 & 0.740 & 0.787 & 0.914 & 0.851 & 0.500\end{array}$ $\begin{array}{lllllllllll}111 & 0.067 & 0.131 & 0.139 & 0.177 & 0.100 & 0.212 & 0.149 & 0.043 & 0.081 & 0.130\end{array}$ 


\begin{tabular}{|c|c|c|c|c|c|c|c|c|c|c|c|}
\hline \multirow[t]{2}{*}{$\begin{array}{l}\text { Locus } \\
\text { name }\end{array}$} & $\begin{array}{l}\text { Allele } \\
\text { size } \\
\text { (bp) }\end{array}$ & \multicolumn{10}{|c|}{ Location } \\
\hline & & 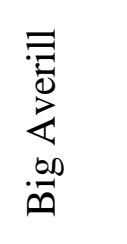 & 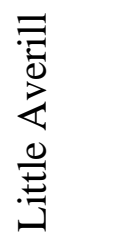 & 当 & 옴 & $\begin{array}{l}\frac{\lambda}{2} \\
\frac{0}{00} \\
0 \\
3 \\
3\end{array}$ & $\sum_{0}^{\pi}$ & 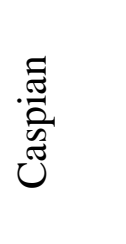 & 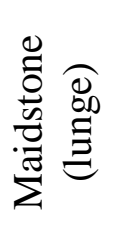 & 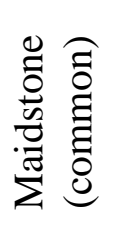 & 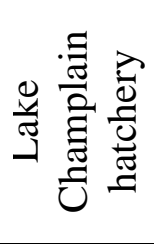 \\
\hline \multirow{21}{*}{ Saz72im } & 99 & & & 0.009 & & & & & & & \\
\hline & 114 & & & & & & 0.010 & & & & \\
\hline & 116 & & & 0.092 & & & 0.153 & & & & \\
\hline & 150 & & & & 0.011 & & & & & & \\
\hline & 170 & & & 0.010 & & & & & & & \\
\hline & 174 & & & 0.051 & 0.152 & 0.012 & & & & 0.021 & \\
\hline & 176 & 0.040 & 0.073 & & 0.098 & 0.049 & & & 0.294 & 0.179 & \\
\hline & 178 & & & 0.020 & & & & & & & \\
\hline & 180 & & & & & & & & & 0.007 & 0.021 \\
\hline & 182 & & 0.024 & & & & & & & 0.007 & \\
\hline & 184 & & & & & & & & 0.059 & 0.036 & \\
\hline & 186 & & & & & 0.012 & & & & & \\
\hline & 188 & 0.010 & & 0.010 & 0.011 & & & & & 0.029 & \\
\hline & 190 & & 0.061 & 0.010 & 0.054 & & 0.031 & 0.033 & 0.088 & 0.093 & \\
\hline & 192 & & 0.049 & 0.020 & 0.033 & & & & & & \\
\hline & 194 & 0.110 & & 0.092 & 0.022 & 0.244 & 0.051 & 0.067 & & 0.036 & 0.404 \\
\hline & 196 & 0.390 & 0.268 & 0.061 & 0.174 & 0.085 & 0.071 & & 0.118 & 0.093 & \\
\hline & 198 & 0.030 & 0.037 & & & & 0.010 & 0.017 & & & 0.011 \\
\hline & 200 & 0.060 & 0.049 & 0.031 & 0.033 & 0.341 & 0.031 & 0.050 & & 0.007 & 0.149 \\
\hline & 202 & 0.080 & 0.061 & 0.020 & 0.022 & 0.085 & 0.020 & & 0.015 & & \\
\hline & 204 & & & & & 0.024 & & & & & \\
\hline
\end{tabular}




\begin{tabular}{|c|c|c|c|c|c|c|c|c|c|c|c|}
\hline \multirow[t]{2}{*}{$\begin{array}{l}\text { Locus } \\
\text { name }\end{array}$} & $\begin{array}{l}\text { Allele } \\
\text { size } \\
\text { (bp) }\end{array}$ & \multicolumn{10}{|c|}{ Location } \\
\hline & & 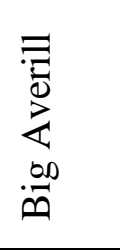 & 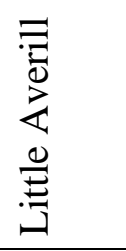 & 当 & 总 & $\begin{array}{l}\frac{2}{0} \\
\frac{0}{00} \\
0 \\
0 \\
3\end{array}$ & 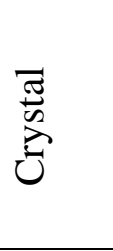 & 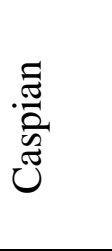 & 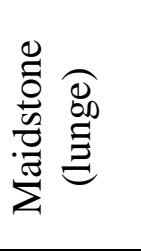 & 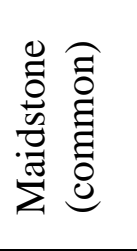 & 总 \\
\hline & 206 & & & & 0.022 & & & & & & \\
\hline & 214 & & & & & & & 0.017 & & & \\
\hline & 216 & & & 0.051 & 0.109 & 0.085 & 0.010 & 0.267 & 0.221 & 0.157 & \\
\hline & 218 & 0.010 & 0.037 & & 0.011 & & 0.092 & 0.017 & & & 0.011 \\
\hline & 220 & & & & & & 0.010 & & 0.029 & 0.036 & \\
\hline & 222 & & 0.024 & & & & & & & & \\
\hline & 224 & & 0.098 & & & & & & & & \\
\hline & 230 & 0.030 & & & & & & 0.033 & & & \\
\hline & 232 & & & & & & & 0.017 & & & \\
\hline & 234 & & & & & & 0.020 & & & & \\
\hline & 236 & 0.220 & 0.220 & 0.520 & 0.022 & 0.037 & 0.490 & 0.483 & 0.176 & 0.243 & 0.128 \\
\hline & 238 & & & & 0.217 & 0.024 & & & & 0.050 & 0.191 \\
\hline & 240 & 0.020 & & 0.010 & & & & & & & 0.085 \\
\hline & 242 & & & & 0.011 & & & & & 0.007 & \\
\hline
\end{tabular}

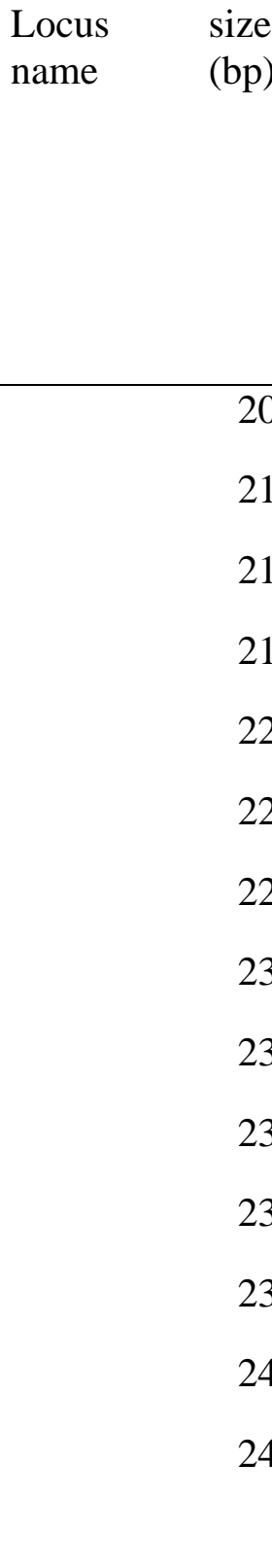

$\begin{array}{llllllllllll}\text { SalE38 } & 100 & 0.784 & 0.635 & 0.827 & 0.766 & 0.778 & 0.794 & 0.829 & 0.357 & 0.542 & 0.590\end{array}$

$\begin{array}{llllllllll}102 & 0.039 & 0.081 & 0.029 & 0.043 & 0.056 & 0.010 & 0.029 & 0.043 & 0.090\end{array}$

$\begin{array}{llllllllll}104 & 0.010 & 0.054 & 0.058 & 0.021 & 0.067 & 0.167 & 0.086 & 0.129 & 0.160\end{array}$

106

110

0.041

0.010

0.010

112

0.010

$114 \quad 0.039$

$0.029 \quad 0.007$ 


\begin{tabular}{|c|c|c|c|c|c|c|c|c|c|c|c|}
\hline \multirow[t]{2}{*}{$\begin{array}{l}\text { Locus } \\
\text { name }\end{array}$} & $\begin{array}{l}\text { Allele } \\
\text { size } \\
\text { (bp) }\end{array}$ & \multicolumn{10}{|c|}{ Location } \\
\hline & & $\begin{array}{l}=7 \\
\sum_{0}^{2} \\
.00 \\
.00\end{array}$ & 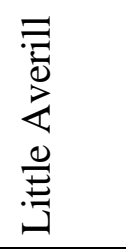 & 裉 & 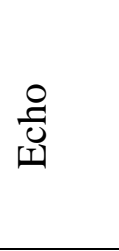 & 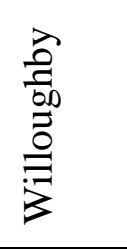 & $\underset{0}{\frac{\pi}{4}}$ & 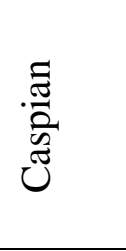 & 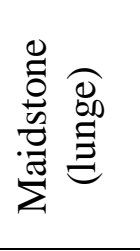 & 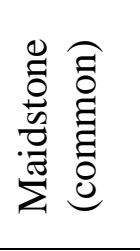 & 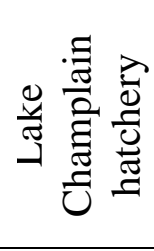 \\
\hline & 116 & 0.039 & & & & & & & & & \\
\hline & 118 & 0.020 & 0.108 & & & & & & 0.043 & 0.028 & 0.130 \\
\hline & 120 & 0.020 & 0.014 & 0.048 & 0.117 & 0.022 & 0.010 & & & 0.007 & 0.040 \\
\hline & 122 & 0.020 & & 0.010 & 0.011 & & & 0.014 & 0.014 & & 0.010 \\
\hline & 124 & & & & 0.011 & & 0.020 & 0.029 & 0.029 & 0.014 & 0.010 \\
\hline & 126 & & 0.041 & & 0.011 & & & & & & 0.010 \\
\hline & 128 & & 0.027 & & & & & & & & \\
\hline & 130 & & & & 0.011 & & & & & & 0.060 \\
\hline & 132 & & & & & 0.011 & & & & & 0.020 \\
\hline & 92 & & & & & & & 0.014 & & & 0.010 \\
\hline & 96 & 0.029 & & 0.019 & 0.011 & 0.067 & & & 0.357 & 0.208 & \\
\hline & 98 & & & 0.010 & & & & & & 0.035 & \\
\hline
\end{tabular}

SalD39 $\quad 142 \quad 0.038$

$\begin{array}{lllllllll}158 & 0.038 & 0.080 & 0.198 & 0.047 & 0.122 & 0.021 & 0.086 & 0.007\end{array}$

$182 \quad 0.010 \quad 0.013$

$\begin{array}{lllllllllll}188 & 0.913 & 0.974 & 0.900 & 0.802 & 0.907 & 0.878 & 0.904 & 0.914 & 0.986 & 0.939\end{array}$

190

192

$0.013 \quad 0.020$

0.047

0.074

0.010

$0.007 \quad 0.051$ 


\section{SUPPLEMENTAL REFERENCES}

Cornuet, J. M., and G. Luikart. 1997. Description and power analysis of two tests for detecting recent population bottlenecks from allele frequency data. Genetics 144:2001-2014.

Earl, D. A., and B. M. von Holdt. 2012. STRUCTURE HARVESTER: a website and program for visualizing STRUCTURE output and implementing the Evanno method. Conservation Genetics Resources 4:359361.

Evanno, G., S. Regnaut, and J. Goudet. 2005. Detecting the number of clusters of individuals using the software structure: a simulation study. Molecular Ecology 14:2611-2620.

Excoffier, L., and H. E. L. Lischer. 2010. Arlequin suite ver 3.5: a new series of programs to perform population genetics analyses under Linux and Windows. Molecular Ecology Resources 10:564-567.

Falush, D., S. Matthew, and J.K. Pritchard. 2003. Inference of population structure using multilocus genotype data: linked loci and correlated allele frequencies. Genetics 164:1567-1587.

Falush, D., S. Matthew, and J. Pritchard. 2007. Inference of population structure using multilocus genotype data: dominant markers and null alleles. Molecular Ecology Notes 7:574-578.

Hubisz, M. J., D. Falush, M. Stephens, and J. K. Pritchard. 2009. Inferring weak population structure with the assistance of sample group information. Molecular Ecology Resources 9:1322-1332.

Jombart, T. 2008. adegenet: a R package for the multivariate analysis of genetic markers. Bioinformatics 24:1403-1405.

Jombart, T., S. Devillard, and F. Balloux. 2010. Discriminant analysis of principal components: a new method for the analysis of genetically structured populations. BMC Genetics [online serial] 11:94.

Pritchard, J. K., M. Stephens, and P. Donnelly. 2000. Inference of population structure using multilocus genotype data. Genetics 155:945-959.

R Development Core Team. 2012. R: a language. R Development Core Team, Vienna. 